\title{
Lou - oder: Publizistik der religiösen Hybris
}

\author{
von Henk Prakke
}

Kurz nachdem der folgende Beitrag über die religiöse Bewegung des niederländischen Aalfischers Louwrens van Voortbuizen in Druck gegangen war, erbielt er im Nachbinein besondere Aktualität. In einer Nachricht auf der Titelseite meldete die auflagenstärkste niederländische Morgenzeitung "De Telegraaf" am 29. März 1968 den Tod Lous, der fünf Tage zuvor im südbelgischen Agimont gestorben sei. Anbänger bätten den erkrankten Lou - so die Zeitung - im Ardennengebiet versteckt und nach seinem Ableben beerdigt. Ortsarzt und Totengräber bestätigten auf Nachfrage diese Meldung. "De Volkskrant" veröffentlichte dazu im Wortlaut die standesamtliche Eintragung. Obne Zweifel: Lou ist tot. Auch die Schriften-Mission der Lou-Anbänger am Hauptbabnhof in Amsterdam wurde eingestellt. Dennoch berichtete dieselbe „Volkskrant", in Agimont halte sich hartnäckig das Gerücht, Lous Sarg sei leer. "Het Parool" besuchte im "Weißen Haus" in Muiden den ,ersten Sohn" von Lou, Joop Halsema (32), vormals Student der Psychologie in Amsterdam, später full-timer in Lou, und erfubr von ibm, Lou sei aufgegangen in seinen Söbnen und Töchtern. Halsema gab über dieselbe Zeitung bekannt, daß Lou am Dienstag, dem 2. April, abends wieder in Amsterdam sprechen werde. Die angekündigte Veranstaltung, der auch das niederländische Fernseben teilweise zu Publizität verbalf, zeigte die zwölf Sohne und Töchter von Lou unter einem großen Banner mit der Aufschrift: Lou lebt! Offenbar soll die Bewegung von einem der Söbne im Geiste Lous weitergefübrt werden.

Damit der Leser es gleich am Anfang weiß: Im Jahre 1972 geht unsere vom Satan beherrschte Welt unter. Naturkatastrophen, Atombomben, radioaktive Verseuchung sind Vorzeichen. Nur wer in Lou (sprich: Lau) den Herrgott selbst erkannt hat, wird gerettet werden und die neue Erde des neuen tausendjährigen Reiches mit beerben. Er ist aber kein zürnender Bußprediger, dieser "Lou de Palingboer van Muiden" (= Lou, der Aalhändler aus Muiden), er ist dem Anschein nach eher ein typischer Kleinbürger. Eine soziologische Studie nennt ihn die "hausbackenste Gottheit", die man sich denken könne.

\section{Biographisches}

Der niederländische Fischer Louwrens van Voorthuizen wurde 1898 in Breezand (nördlich von Alkmaar) in einer strenggläubigen Bauernfamilie geboren. Im Jahre 1927 erlebte er (nach der eigenen Formulierung) den Untergang seines menschlichen ichs (mit kleinem i), worauf das göttliche Ich (mit großem I) die Herrschaft antrat über das Geschöpf, das Lou hieß. "Gott hat das Nichts genommen, das Geschöpf, und kann in diesem Nichts alles sein."

Prof. Dr. Henk Prakke (Meppen-Zweeloo/Niederlande) ist Direktor des Instituts für Publizistik der Universität Münster. 
1950 begann Lou in der Offentlichkeit zu predigen: Ich predige Jesus Christus leiblich, seinen Auferstehungsleib mit seinem neuen Namen, und das ist Lou.

Nach und nach entstand aus einigen hundert "Lou-Menschen ", wie sie sich nennen, eine Gruppe von Anhängern. Lous Ehefrau, die einige glückliche Jahre mit ihm zusammenlebte, darunter zwei auf gemeinsamer Aalfischereifahrt auf dem Ijsselmeer, trennte sich von ihm, als die Vorstellung, Jesus zu sein, über ihn kam. Heute lebt er mit einer seiner ersten Anhängerinnen zusammen: Mientje, — sie hat mit ihrer Begeisterung möglicherweise das meiste zum Lou-Kult beigesteuert.

Schon als Kind wollte Lou nicht mit seinen Eltern zur Kirche gehen. Eine Zeitlang gehörte er zu den „Onderdompelaars“, zur "Pfingstgemeinde“. Nach Lous Uberzeugung soll im Jahre 1520 der letzte Mensch, der vom Heiligen Geist überkommen war, ausgerottet worden sein. Damit hatte Satan sein Spiel gewonnen. Ohne den Heiligen Geist wurde sogar die Bibel ein Werkzeug des Teufels. Bibel und Theologie und überhaupt jede Intellektualität sind von Ubel. In unserer Gegenwart ist dann also Lou erschienen als Erfüller Jesu Christi für diejenigen, die seine Predigt annehmen und Söhne und Töchter Gottes werden wollen. Die Vernichtung des Satans ist Lous Aufgabe, die Kinder Gottes werden durch die Liebe der Gewalt des Todes entzogen; das bedeutet Unsterblichkeit auch für die gläubigen Anhänger. Der Untergang des Satans ist nicht mehr aufzuhalten. So etwa kann man die in verschleierter Sprache gehaltene Botschaft kurz zusammenfassen, mit der Lou als religiöser Führer an die Offentlichkeit tritt.

Zusamnıen mit einer kleinen Zahl von Anhängern wohnt Lou in seinem „Weißen Haus" in Muiderberg. Jeder Interessent ist ihm dort willkommen. Einmal im Monat predigt er in Amsterdam, und zwar jetzt im Versammlungslokal "Frascatic, häufig mittels eines Tonbandvortrages. Seine Schüler unterhalten sich dort mit einer anscheinend immer diskussionsfreudigen Hörerschaft.

Innerhalb der Gruppe entwickelte sich eine gewisse Hierarchie: An der Spitze stehen Lou und Mientje, dann folgen 12 (= $4 \times 3$ : magische Zahlen) "metgezellinnen“ (Gefährtinnen), die die Gabe des „direkten Wissens" haben, schließlich 50 „Söhne von Lou“, die der Welt seine Heilsbotschaft zu verkünden haben. Lou spricht jetzt „mit dem Mund seiner Söhne“.

Im Jahre 1956 gab es gerichtliche Schwierigkeiten, als das Kind eines Lou-gläubigen Ehepaares krank wurde und starb. Auf Lou vertrauend hatte man keinen Arzt hinzugezogen. Lou wurde als Zeuge vor Gericht zitiert. Er erschien nicht, indem er sich auf seine Göttlichkeit berief: Gott läßt sich nicht zitieren. Wäre er gebeten worden, so wäre er sicherlich gekommen.

$\mathrm{Zu}$ jener Zeit schien Lou auch seinen Theologen gefunden zu haben. Der Amsterdamer Archivar Ton Haentjens Dekker stellte in seiner Schrift „Rumoer om Lou de Palingboer" in einer eher auf Intellektuelle zugeschnittenen Sprache Lou als einen großen Mystiker dar. Sobald dieser aber immer mehr die Messias-Rolle aufgab und sich für Gott selbst zu halten begann, distanzierte Dekker sich wieder von ihm. Als vor kurzer Zeit Lou mit Mientje wieder einmal in Amsterdam auftrat und bei dieser Gelegenheit auch im Fernsehen interviewt wurde, zeigten beide, obwohl man bei Lou Spuren des Alters glaubte feststellen zu können, ihre unverminderte Gewißheit über Lous Berufung.

\section{Die Botschaft}

Der Soziologe A. E. Bayer, der seit 1955 die Lou-Bewegung in partizipierender Wahrnehmung besser kennengelernt hat als irgendein anderer Außenstehender und 
den Entwicklungsgang der Gruppe aufmerksam weiterverfolgte, hat einmal versucht, Lous Botschaft zusammenzufassen.

Es ist sehr verständlich, so resümiert Bayer ${ }^{1}$, wenn ein Fremder, der direkt von der Nes-Straße ins „Frascati“ eintritt, dort Lous Anhänger sprechen hört und nachher in die Diskussion hineingezogen wird, sich zu fragen beginnt, ob er selbst verrückt geworden ist oder ob alle anderen es sind. Die Sprache, in der die Botschaft Lous und seiner Söhne gehalten ist, klingt dunkel. Lou ist bei aller Bibelfestigkeit kein gebildeter Mann im Sinne einer schulischen Erziehung zur Bildung. Wie seine Anhänger vor Bayer wiederholt ausgeführt haben, könne er nicht einmal lesen oder schreiben. Wer sich die Mühe machen will, Lou und seine Schüler zu verstehen, muß vor allem auf einige Merkwürdigkeiten in ihrem Sprachschatz Rücksicht nehmen, die zunächst irritieren. Ein Teil des offensichtlichen Mangels an Logik kann durch eine Korrektur des dort üblichen Wortgebrauchs berichtigt werden. Lou bedient sich bisweilen mit Vorliebe eines Wortschatzes, der viele Elemente aus der alten niederländischen „Staten-Ǔbersetzung“ der Bibel (aus dem 17. Jahrhundert) enthält. Daneben gebraucht er Redensarten, die früher in extrem strenggläubigen Sekten vorkamen und die bisweilen von ekstatischen Gemeinschaften, etwa der Pfingstgemeinde, übernommen wurden. Wenn Lou über den "gestürzten Buchstaben " spricht, bedeutet das, daß die Bibel ihre ursprüngliche Kraft für den Menschen verloren habe und $\mathrm{zu}$ einem Buch wie alle anderen Bücher geworden sei. Unsere Generation lebt, nach Lou, „unter den Zeichen“, d. h. das Ende der Welt ist angekündigt. Schließlich prägt Lou gelegentlich auch Neuformulierungen. Seine "Söhne“ halten sich im allgemeinen an seinen Sprachgebrauch.

Lou hat, ich referiere weiter die Darstellung Bayers, wiederholt erklärt, daß er nichts Neues verkünde. Das trifft zu. Abgesehen von seiner Prätention, daß gerade er, Lou Voorthuizen, der Besieger des Satans sein werde, kommen viele seiner Thesen schon in den mystischen Schulen der Zeit des jungen Christentums vor, aber auch in östlichen Religionen und sogar bei modernen Philosophen. Dieses macht zwei Annahmen möglich: Lou könnte entweder ein Betrüger sein, der einen religiösphilosophischen Cocktail zusammengemischt hat, um ihn - und sich selbst im Mittelpunkt - als Heilslehre anzubieten, oder: Lou ist ein Mensch mit einer gewissen Begabung zum authentisch-religiösen Erleben, der mehr oder weniger instinktiv und während endloser Gespräche, die er oft mit höher Gebildeten führt, gerade jene Elemente heraussucht und für sich behalten hat, die als Bausteine für seine Heilslehre dienen könnten. Wenn wir von einer Entscheidung zwischen diesen beiden Möglichkeiten absehen, bleibt in jedem Falle noch eine ansehnliche Leistung übrig: Eine Lehre, die in sich selbst verhältnismäßig logisch zurechtgezimmert ist, die aber in einigen wichtigen Punkten philosophische Achilles-Fersen aufweist.

Lou sieht den „Stoff“ als die Schöpfung Gottes, als die jungfräuliche Substanz, um die Gott und der Satan kämpfen. Dieser sein Lehrsatz stimmt mit Auffassungen von Gnostikern in den ersten Jahrhunderten nach Christus überein. Der Satan, der Gott gleich sein wollte, praktiziert seinen Griff nach dem Stoff mit Hilfe des Intellekts, der bei Lou „der Begriff“ oder „das Denken “ genannt wird. Unausgesetzt warnt er vor jenen, „die es so gut wissen“, und vor jenen, die meinen, Gott zu haben, wenn sie einen Gottesbegriff haben, so zum Beispiel mit dieser Wendung: Weil Israel es so gut wußte, ging es unter. Man kann es nicht wissen. Ein jeglicher soll den Weg gehen, den er nicht gewußt hat; es ist trotzdem der Weg, auf dem die Armen im Geiste nicht irren können.

„Die Gesetzmäßigkeit des Todes liegt außerhalb Gottes“, sagt Lou. Tot sein heißt 
außerhalb Gottes sein. Gott ist die Erlösung vom Tode. Dieser Lehrsatz erinnert an Auffassungen aus dem Buddhismus, nach dem Pali-Kanon, wonach Mara, der Böse, das Reich des Todes regiert und die Erlösung von seiner Macht notwendig zur Erlösung vom Tode führen muß. Wie kann der Böse besiegt werden? Durch die Kraft des Glaubens. In dem Maße, in dem jemand glaubt, wird er Kraft finden. Ferner kehrt in unterschiedlichen Formulierungen der Satz wieder: „Die Verborgenheit aus dem Nichts offenbart sich. "Hinter dieser Betonung der Negation als Ausweg steht die Traditon der Mystik aller Länder und Zeiten. (Marcion, Angelus Silesius, Jakob Boehme und eine ganze Reihe von Schriften aus dem Mahayana-Buddhismus.) Von der Bibel bezeugt Lou: Der Buchstabe ist gestürzt. Es ist eine Unmöglichkeit, vom Buchstaben aus zu Christus zu gelangen. Hier muß man an Außerungen der Sufi im Islam denken, etwa an Mevlana von Konya, dann an die chinesisch-japanischen Zen-Schulen, wo ein Großmeister sagt: "Wirf den Verstand vor die Hunde." Man wird aber auch an Strömungen in der westlichen Philosophie erinnert, etwa an den Vitalismus von Bergson und an die Existenzialisten. Das ganze Klima dessen, was Lou lehrt, besonders sein Kampf gegen das „Denken“, erinnert an Ludwig Klages: „Der Geist als Widersacher der Seele“. Soweit die Ausführungen Bayers über den Kern der Lou'schen Botschaft.

\section{Die publizistischen Mittel}

Lou bedient sich der für die kleine Gruppe typischen publizistischen Mittel, vornehmlich der Rede und der Diskussion. In der Schrift-Publizistik seiner Gruppe gab es in der ersten Zeit hektographierte Reden für Werbezwecke, dann eine Zeitschrift, die anfangs "De Pyramide" hieß. Heute erscheint sie unter dem lapidaren Titel "Maandblad LOU“ (Monatsblatt LOU); die Auflage beträgt 30000 Exemplare. Mit einer deutschen Ausgabe wird in gelegentlichen Kampagnen missioniert - z. B. in Münster/Westfalen auf dem Domplatz oder vor dem Bahnhof. Es existiert offenbar auch französisches und englisches Werbematerial; ein Zeuge spricht von Ubersetzungen in die ungarische Sprache. Der Fixierung, aber auch dem Vortrag von LouReden dient des öfteren das Tonband. Eine Schallplatte mit „Verklaringen " von Lou und dem "Roeplied" von Mientje soll im Schallplattenhandel zu haben sein.

Die größte Publizität wurde anläßlich des erwähnten Gerichtsverfahrens im Jahre 1956 erzielt; Lou wurde von den modernen Massenmedien entdeckt, die sich seither besonders bei Streitigkeiten innerhalb der Gruppe in die Berichterstattung einschalten und dann und wann einen Renegaten zu Wort kommen lassen. (Kommentar von Lous Sekretärin zu diesem Verfahren: "Journalisten gleichen Hunden, die in Mülltonnen wühlen.") Im Fernsehen ist Lou jedenfalls zweimal zu Wort gekommen: 1962 und 1967.

Zum Mittel der Diskussion sei noch gesagt, daß Lous Anhängerschaft auch Treffen anderer religiöser Gruppen, z. B. Zeltmissionen, dazu benutzt, um den Besuchern dieser Veranstaltungen ihre eigene Sache vorzutragen; vor den Zelteingängen versucht man, Interessenten abzufangen.

\section{Hörerschaft und Wirkung}

So wie Lou in seinem Außeren, seinem Benehmen und seiner Sprache ein gewöhnlicher Mann aus dem Volke ist, so gibt sich auch seine Anhängerschaft. In der Pause seiner Diskussionsabende pflegt Lou sein Bier zu trinken und im Kreise seiner treuesten Anhänger eine Zigarre zu rauchen. Unter die Hörerschaft mag sich immer wieder der eine oder andere Intellektuelle mischen, der allgemeine Eindruck aber ist, 
daß es sich bei seinen Hörern um freundliche Leute aus dem Volke handelt, mittleren Alters oder jünger, unter ihnen auch eine Anzahl junger Menschen, nur wenig mehr Frauen als Männer. Lous ruhige, selbstsichere Redeweise findet offenbar Anklang. Seine dunkle Wortgewalt, - er spricht über eine Stunde lang ohne Manuskript oder Notiz, - ruft bei der dafür aufgeschlossenen Hörerschaft den Eindruck der Tiefsinnigkeit hervor. Ubrigens zieht er nicht nur die Aufgeschlossenen an. Gerade in Amsterdam findet sich für religiöse Fragen immer wieder ein diskutier-freudiges Publikum: Niederländer seien, so hat man im Scherz gesagt, durchweg halbe Theologen. Gelegentlich allerdings geriet eine Lou-Veranstaltung auch zu einem Happening (avant la lettre). "Geistlicher Rock-and-Roll rings um Lou" schrieb eine Zeitung schon 1956 über eine Lou-Diskussion im Amsterdamer Hotel Krasnapolsky über die Frage „Kann Lou der wiedergeborene Christus sein?" Fast tausend Menschen aus allen Schichten fanden sich hier zu einer von Zwischenrufen und Lachsalven unterbrochenen Sitzung zusammen, wobei man in Rechnung stellen muß, daß Lou über eine gewisse volkstümliche Schlagfertigkeit verfügt. Er erzählt gern Anekdoten, z. B. über einen Theologen, den er einst in die Enge getrieben hat. Unter seiner Ausstrahlung ergab sich jedenfalls ein merkwürdiges Gemisch aus hysterischer Erwartung und amsterdamschem Witz.

Aus Wortgefechten und Veranstaltungen solcher Art bildete sich eine ergebene feste Anhängerschaft von 400 bis 500 Menschen. Zeitungsberichte über die Lou-Bewegung erwähnen Gemeinden seiner Richtung auch im Ausland, so in Australien, Belgien, Deutschland und Ungarn. Genaue Beobachter seiner Gruppe meinen allerdings, daß die Entwicklung seit Jahren stagniere. Die festen Anhänger sollen zum Teil völlig säkularisierten Schichten entstammen; daneben aber gibt es viele Suchende. $\mathrm{Zu}$ einem zufälligen Kontakt plus Neugierde kommt manchmal die plötzliche Erleuchtung hinzu, daß Lou „die Wahrheit" sein könnte. Dem kirchenentwöhnten Zuhörer sagt die nüchterne Atmosphäre zu. Es gibt bei Lou kein Tremendum, kein Fascinans, kein Mysteriosum. Seine wiederholten Angriffe gegen die offiziellen Kirchen, die Bibel und die Wissenschaft („Denken ist satanisch, Wissen ist ,das göttliche Wissen“.) entsprechen manchen angestauten Feindseligkeitsgefühlen.

In Anlehnung an ein Wort von Alfred Weber über den Islam (eine Religion des religiösen Minimums bei einem Maximum von äußerer Ritualität) könnte man bei der Lou-Bewegung von einer Religion des religiösen und rituellen Minimums sprechen. Nur der Mundkuß als Bruderkuß ist allgemeiner Brauch. Das Geheimnis der Wirkung des religiösen Führers Lou findet man übrigens schon bei Aristoteles angeführt in der Feststellung, daß die Gebildeten allgemein und abstrakt sprechen, die Ungebildeten dagegen sich an das halten, was ihnen bekannt ist und was ihnen nahe liegt. Darum pflegten die Ungebildeten beim breiten Volk einen größeren Eindruck zu machen. Lou kokettiert gern mit seiner Ungebildetheit.

Die Geborgenheit in der kleinen Gemeinschaft, in der es im Unterschied zu den offiziellen Kirchen keine Distanz zwischen Prediger und Gemeinde gibt, wirkt auf viele Interessenten attraktiv. Darüber hinaus gilt die Bewegung ihren gläubigen Anhängern als Waffe gegen Krankheit und Tod. Dabei scheint die Pseudo-Erfüllung der Erwartung eine Rolle zu spielen, daß man durch Lou von der Angst vor Atombombe und radioaktiver Strahlung befreit werden könne.

\section{Wissenschaftliche Beobachtung}

Soziologen und Sozialpsychologen haben das Phänomen des religiösen Führers Lou ernst genommen. Man vergleiche dazu etwa die Arbeit von Bayer und Köbben². 
Die Theologen dagegen haben sich bisher nur in recht kritischen Verurteilungen geäußert. Buskes z. B. meint, daß es sich um einen Exzeß handele. Die Botschaft Lous sei, religiös bewertet, nur lächerlich; das Traurige sei aber auch jetzt wieder, $\mathrm{daß}$ eine beträchtliche Zahl unausgeglichener Menschen in den Bann eines solchen Mannes gerate ${ }^{3}$. Teeuwen verwies Lou schon sehr früh in die Randgebiete der Psychopathologie: als ein merkwürdiges Beispiel von Verblendung primitiver, ungebildeter Geister, die dennoch über zerrüttete Seelen emotionale Herrschaft gewinnen könnten, über Seelen, die weder durch Bibelkenntnis noch durch besondere Intelligenz Möglichkeiten der Gegenwehr haben. Kok hält den Nonsens, der von Lou in holprigen Sätzen dem Publikum vorgelegt werde, für erschreckend, für empörende, düstere Schwafelei.

Dieser Protest gegen die Hybris der Lou'schen Prätention mag verständlich sein, die Frage aber, ob die ganze Lou-Bewegung, wie der niederländische Schriftsteller Harry Mulisch vorgeschlagen hat, nur „Futter für die Psychologen“ sei, bleibt weiterhin ungelöst. Wer sich für Religionspublizistik interessiert, - Religionspublizistik verstanden als die "Lehre der Erfahrung des Heiligen in ihrer Wirkung auf das gesellschaftliche Zwiegespräch", - möchte Bayers Blickwinkel, der die Mystik als Kategorie einbezieht, einmal unvoreingenommen durch Theologen untersucht sehen. Der oben angeführte Katalog der publizistischen Mittel, die von den Lou-Menschen angewendet werden, reicht nicht aus, um das Phänomen Lou und seine Wirkung in seinem Wesen $z u$ verstehen. Die Soziologie deutet dies an, indem sie vom Typ des charismatischen Führers spricht. Für seine ihm ergebene Gefolgschaft verfügt Lou gewiß über dieses Charisma. Wie vermittelt es sich? Bayer und Köbben urteilen, daß Lou mit seinem durchdringenden Blick und seinen scharfen Zügen gewiß kein Durchschnittsmensch sei. Er mache den Eindruck, seiner Sache vollkommen sicher zu sein, wirke ruhig, spreche meistens auch ruhig, aber man spüre doch eine kaum bezwungene ungestüme Emotionalität unter der Oberfläche.

Hier stehen Fragezeichen für die Religionspublizistik. Sie hätte sich, mit Hilfe der Theologen, einmal mit Lou als Beispielfall zu befassen. Beim Begriff Mystik assoziiert man heute meist Vorstellungen wie: außerhalb der Welt, Zurückgezogenheit eines Klosters, Askese, Meditation, Kontemplation, eventuell Ekstase. Erwägt man aber die Möglichkeit einer weltbezogenen, gewissermaßen säkularisierten Mystik, dann kann die Hybris der Grenzverwischung zwischen Subjekt und Objekt, wodurch Mensch und Welt oder Mensch und Gott eins werden, für das gesellschaftliche Zwiegespräch nicht ohne Folgen sein. Die Entfaltung eines publizistisch wirksam werdenden Charismas läßt sich wahrscheinlich auch in unserer der rechenhaftigen Rationalität zugeneigten Zeit nicht durch Messen und Zählen feststellen, wenngleich wir methodisch durchaus in der Lage sind, auch bestimmte Qualitäten quantitativ faßbar zu machen. Lous Qualität auf diesem Gebiet ist jedoch nicht durch die Zahl seiner Anhänger $\mathrm{zu}$ beschreiben. Sollte es nicht dennoch möglich sein, an einem so kleinen, überschaubaren Exempel Erkenntnisse über die religiöse Determinante eines mystisch oder pseudo-mystisch infizierten Kommunikationssystems zu gewinnen und dabei dessen Verknüpfung mit dem größeren Kommunikationssystem einer Gesamtgesellschaft aufzudecken?

Anmerkungen:

1. A. E. Bayer: Lou Voorthuizen daagt de dood uit, in: „Vrij Nederland“, Amsterdam, Ausg. vom 10. März 1962.

2. A. E. Bayer und A. J.F. Köbben: Leider en volgelingen in een religieuze groep. Lou 
en zijn aanhangers, in: M. Mulder e. a. (Hrsg.): Mensen, groepen, organisaties. Speurwerk in sociale psychologie, Assen 1963, Bd. I, S. $109 \mathrm{ff}$. - Vgl. ferner

A. E. Bayer und A. J. F. Köbben: De Lou-Mensen: Sociografie van een afwijkende groep, in: "Sociologisch Bulletin“, Utrecht/s'Gravenhage 1955, S. $77 \mathrm{ff}$.

3. nach „Het Vrije Volk“, Amsterdam, Ausg. vom 12. Juni 1954. Weitere Aufsätze oder Berichte in Zeitungen und Zeitschriften: "Algemeen Handelsblad", Amsterdam, 25. 9. 1959. "Nieuwe Rotterdamsche Courant" 24. 8. 1959. "Het Vrije Volk “ 27. 2.1962.

\section{S U M M A R Y}

Lou, the Dutch founder of a religious sect (actually: Louwrens van Voorthuizen, born in 1898 in Breezand near Alkmaar and by profession a fisherman) considers himself to be a new incarnation of Jesus Christ. He says that he preaches Jesus Christ physically. $\mathrm{He}$ has preached openly since 1950. The rather small community of "Lou-advocates" that has gathered around him is waiting the end of the Satan-dominated world in 1972. Satan, as they see it, manifests himself especially through all intellectualism. The Bible itself has become the devil's tool; as has, of course, all theology. The group has its headquarters in Lou's "White House" in Muiderberg. He preaches once a month in Amsterdam. Lou has managed to get a remarkable amount of publicity for his group in Holland and the neighboring countries at little expense to himself. He sells a monthly called "Maandblad Lou" and his subscription drives penetrate even far into West Germany. The discussion-happy Dutchmen show quite a bit of interest in the Lou group, but after having heard them, they wonder just who is out of his mind: Lou or his audience. Lou gains a fair amount of publicity by featuring the "common ordinary little man on the street" who carries more interest value for the people of his own wage bracket than do the subtle intellectuals. Until now it has been more the sociologist than the theologian who has undertaken to describe the Lou phenomenon. However, those concerned with religious publicity have something of interest to learn, namely, how a small group like this whose members seem to be mystically endowed makes use of communications media. Someone should delve deeper into these publicity methods but this time in conjunction with a theologian.

\section{RESUMEN}

Lou, fundador de una secta holandesa ( $\mathrm{Su}$ verdadero nombre es Louwrens van Voorthuizen, nacido en 1898 en Breezand, cerca de Alkmaar, Holanda, de profesión pescador), se considera una nueva encarnación de Jesucristo. Dice: „Yo predico a Jesucristo en persona". Predica públicamente desde 1950. Ha reunido en torno a sí una comunidad relativamente exigua de „Hombres de Lou“. Esperan que, en el año 1972, tendrá lugar la caída de este mundo dominado por Satanás. Este se manifiesta sobre todo a través de los intelectuales. La misma Biblia se ha convertido en un instrumento de Satanás y, naturalmente, también la teología. El Centro de este grupo se halla en la „Casa blanca“ que Lou posee en Muideberg. Una vez al mes, predica en Amsterdam. Sin muchos gastos, Lou ha conseguido dar una gran publicidad a su grupo, tanto en Holanda como en los paises vecinos. La revista „Maandblad LOU“ (Revista Mensual de LOU) se ofrece hasta en la Alemania Occidental. Los holandeses, muy interesados en discusiones teológicas, prestan gran interés al Grupo de Lou, pero, cuando le oyen, se preguntan: ¿Quién es el loco, Lou o el que le escucha? Lou debe su publicidad al „ignorante hombre modesto del pueblo“, para el que tiene más importancia uno de su propio estado que el intelectual refinado. Hasta el momento, los sociólogos han mostrado más interés que los teólogos por el fenómeno Lou. También aquí puede hallar el periodismo religioso un interesante campo de observatión: el sistema de comunicación de un grupo pequeño que, sin duda alguna, está impregnado de un componente místico. Una investigación periodística sobre Lou, en colaboración con teólogos, sería muy interessante y debería realizarse. 